\title{
Meditation reduces brain activity in the default mode network in children with cancer
}

\author{
Aneesh Hehr ${ }^{1}$, Allesandra Iadipaolo ${ }^{2}$, Austin Morales ${ }^{1}$, Cindy Cohen ${ }^{3}$, Jeffrey Taub ${ }^{4}$, \\ Felicity Harper ${ }^{5}$, Elimelech Goldberg ${ }^{3}$, Martin Bluth ${ }^{1}$, Christine Rabinak ${ }^{2}$, and Hilary \\ Marusak $^{1}$ \\ ${ }^{1}$ Wayne State University \\ ${ }^{2}$ Wayne State University - Eugene Applebaum College of Pharmacy and Health Sciences \\ ${ }^{3}$ Kids Kicking Cancer \\ ${ }^{4}$ Children's Hospital of Michigan \\ ${ }^{5}$ Karmanos Cancer Institute
}

January 28, 2022

\begin{abstract}
Background: Mounting evidence demonstrates that meditation can lower pain and emotional distress in adults, and more recently, in children. Meditation may be an effective form of emotion regulation in children because it is easily accessible and more easily done across a variety of settings in which toys may not be available, for e.g., settings requiring a "clean" environment (e.g., surgical prep). Further, neuroimaging studies in adults suggest that meditation techniques are neurobiologically distinct from other forms of emotion regulation, such as distraction, that rely on prefrontal control mechanisms, which are underdeveloped in youth. Rather, meditation techniques may not rely on "top-down" prefrontal control and may therefore be utilized across the lifespan. Procedure: We examined neural activation in children with cancer, who experience significant distress. During neuroimaging, children viewed distress-inducing video clips while using martial arts-based meditation (focused attention, mindful acceptance) or non-meditation (distraction) emotion regulation techniques. In a third condition (control), participants passively viewed the video clip. Results: We found that meditation techniques were associated with lower activation in default mode network (DMN) regions, including the medial frontal cortex, precuneus, and posterior cingulate cortex, compared to the control condition. Additionally, we found evidence that meditation techniques may be more effective for modulating DMN activity than distraction. There were no differences in self-reported distress ratings between conditions. Conclusion: Together, these findings suggest that martial arts-based meditation modulates negative self-referential processing associated with the DMN, and may have implications for the management of pediatric pain and negative emotion.
\end{abstract}

\section{Introduction}

The ability to regulate emotion develops across the first two decades of life, corresponding with maturational changes in brain networks supporting emotion processing and self-regulation. ${ }^{1}$ Emotion regulation is central to developmental outcomes. ${ }^{2-4}$ Deficits in emotion regulation can increase risk of mental disorders in adulthood (e.g., depression ${ }^{5}$ ). Therefore, strategies that are effective for bolstering emotion regulation during childhood may have long term benefits, such as ameliorating adverse outcomes during adulthood. ${ }^{6}$ Emotion regulation strategies that involve forms of mindfulness and meditation originate from ancient practices. ${ }^{7}$ Meditation refers to an umbrella of mental practices that involve the monitoring and regulation of attention and emotion. ${ }^{8,9}$ Mindfulness is a form of meditation that involves focusing attention to and accepting thoughts and emotions in the present. ${ }^{10}$ There are also individual differences in the tendency towards mindfulness (i.e., trait mindfulness) that can change over time through deliberate meditation practice. ${ }^{11}$ Meditation 
techniques are now integrated into established treatments for psychological disorders involving emotion dysregulation, including depression. ${ }^{12}$ Meditation programs have been shown to be effective for reducing stress, anxiety, depression, and pain among clinical ${ }^{13}$ and non-clinical ${ }^{14}$ adult populations. They show promise for reducing disease- and treatment-related distress among patients with chronic conditions, such as cancer. ${ }^{15}$ Active engagement in meditation has also been shown to lower self-reported pain and negative emotion. ${ }^{16,17}$

Although most research has been conducted in adults, emerging evidence suggests that mindfulness and meditation are beneficial for children. A meta-analysis of 33 randomized controlled trials reported benefits of meditation programs on attention, depression, and stress among clinical and non-clinical pediatric samples. ${ }^{18}$ Mindfulness and meditation-based programs are popular in school settings, ${ }^{19}$ and increasingly used to help children cope with stressful experiences, such as chronic conditions (e.g., cancer) ${ }^{20,21}$ or trauma. ${ }^{22}$ Meditation as an emotion regulation strategy is promising for preventing mental disorders, given that nearly half of all mental disorders begin during childhood and adolescence. ${ }^{23}$ However, the neural mechanisms supporting meditation as an emotion regulation strategy in children remain unclear. Insight on these mechanisms could help validate and improve interventions for at-risk pediatric populations exposed to high stress and trauma.

Meditation strategies are distinct from other forms of emotion regulation, such as distraction. Rather than controlling attention away from emotional experiences, meditation strategies involve noticing and accepting emotional reactions as they arise. ${ }^{8}$ Meditation also appears to be neurobiologically distinct from other forms of emotion regulation that rely on 'top down' modulation of emotional responses. More 'cognitive' forms of emotion regulation, such as distraction, commonly engage brain regions in the central executive network $(\mathrm{CEN})$, with core nodes in the dorsolateral prefrontal cortex and the parietal cortex. ${ }^{24,25}$ Meditation emotion regulation strategies do not appear to engage the CEN. Rather, these techniques have been shown to reduce activation in regions of the default mode network (DMN), including the medial frontal cortex (MFC), posterior cingulate cortex, and precuneus, during deliberate meditation and exposure to aversive stimuli. ${ }^{16,17,26}$ The DMN is associated with self-referential thought and depressive rumination. ${ }^{27,28}$ Prior research suggests that depressed individuals fail to downregulate DMN activity when exposed to negative emotional stimuli. ${ }^{29}$ Taken together, existing research suggests that meditation can modulate DMN activity, and these effects do not rely on 'top-down' control mechanisms. This is important because children might not be able to employ complex regulatory strategies due to underdevelopment of the CEN. ${ }^{30,31}$ Further, simple meditation strategies, such as focused attention to the breath, may be more accessible to children in stressful situations.

To our knowledge, only two functional magnetic resonance imaging (fMRI) studies have examined the neural correlates of meditation training in children. These studies report lower amygdala response to fearful faces and lower resting-state functional connectivity between the DMN and CEN in children (mean age = 11.75 years) following an 8-week mindfulness-based school program relative to a control program. ${ }^{32,33}$ These finding support the notion that meditation can modulate brain activity (e.g., DMN) in children. These patterns are consistent with our prior fMRI study showing that more trait mindful youth spent less time in a dynamic functional connectivity state characterized by higher DMN-CEN connectivity. ${ }^{34}$ However, no studies to our knowledge have examined neural activity in children actively engaged in meditation emotion regulation.

To address this gap, we examined the within-subjects effects of meditation on brain activity in a sample of children with cancer, who experience significant disease and treatment-related distress. ${ }^{35}$ Participants received minimal meditation training $(4 \mathrm{hrs})$ through a well-established martial arts-based meditation program ${ }^{21,36}$ prior to undergoing an adapted version of an established fMRI emotion regulation task in the scanner. We compared two meditation emotion regulation techniques (i.e., focused attention and mindful acceptance) with a control condition (i.e., passive viewing) and with a non-meditation emotion technique (i.e., distraction). Participants were asked to engage in one of the four conditions while they were exposed to aversive video clips (e.g., a child receiving an injection), and rated their negative emotion after each trial. This design allowed us to test (1) whether meditation techniques can reduce brain activity in the DMN, and (2) whether meditation emotion regulation techniques are more effective than a non-meditation technique - distraction - at reducing DMN activity. We also (3) compared brain activation during the two meditation techniques, based on evidence that different meditation techniques have different effects on brain activity. ${ }^{37}$ 


\section{Methods and Materials}

\subsection{Participants}

This preliminary study reports on 12 childhood cancer patients or survivors (ages 5-17, 5 female) recruited from the Children's Hospital of Michigan Hematology/Oncology clinic (Detroit, MI) and from local cancer support groups and organizations (e.g., Kids Kicking Cancer, Gilda's Club of Metro Detroit). Data were collected from August 2017 through January 2019 as a part of a larger prospective study examining the effects of a martial arts-based program, Kids Kicking Cancer (www.kidskickingcancer.org), on pain, emotional distress, and health-related quality of life among children with cancer (Supplemental Methods 1.1). Eligible participants were ages 5-17 (inclusive), English-speaking, and previously diagnosed with any form of pediatric cancer that did not include the central nervous system. Youth were excluded if they had MRI contraindications (e.g., claustrophobia, braces, non-MRI compatible port), major sensory impairments (e.g., severe vison loss), comorbid neurological disorders (e.g., epilepsy), gross neuropathologies (e.g., ventriculomegaly), pervasive developmental disorders, or other severe psychopathology (e.g., schizophrenia). The study was approved by the Wayne State University Institutional Review Board. Written informed consent and assent were obtained from participating primary caregivers (i.e., parent or legal guardian) and youth, respectively. Participant demographics and clinical characteristics are provided in Table 1 .

\subsection{Questionnaire measures}

Prior to the MRI scan, youth were assisted by a trained member of the research staff in completing standardized self-report measures of anxiety and trait mindfulness.

Anxiety symptoms: Youth reported on their anxiety symptoms using the 41-item Screen for Child AnxietyRelated Emotional Disorders (SCARED) ${ }^{38}$ shows good reliability, as measured via internal consistency and test-retest reliability. The SCARED demonstrates good discriminate validity between anxiety and nonanxiety disorders. ${ }^{39,40}$ Total possible SCARED scores range from 0-82, with scores of [?] 25 recommended for differentiating anxious from nonanxious youth. In the present sample, $25 \%$ of participants exceeded this threshold for detecting anxiety.

Trait mindfulness: Youth completed the Child and Adolescent Mindfulness Measure (CAMM), a 10-item measure of trait mindfulness. ${ }^{41}$ Possible scores range from $0-40$, with higher scores indicating higher mindfulness. The CAMM shows adequate internal consistency (Cronbach's alpha $=0.81-0.88$ ), reliability, and validity in youth samples. ${ }^{41,42}$

\subsection{Emotion regulation task}

Participants completed an adapted emotion regulation task ${ }^{43,44}$ during fMRI scanning. During the task (Figure 1 ), participants viewed distress-inducing stimuli and rated their current emotional distress after each trial. Participants rated their distress on a 1-6 visual analog scale (VAS; $1=$ "No distress at all", $6=$ "Worst possible distress") using an adapted version of the FACES scale. ${ }^{45,46}$ Task stimuli consisted of ten validated $30 \mathrm{~s}$ video clip vignettes depicting various realistic, salient stressors (e.g., child receiving an injection) that have been shown to induce transient distress in children. ${ }^{47}$ Negative stimuli were used to test for differences in brain response to distress-inducing stimuli. Participants were explicitly instructed to pay attention to the movie as if it were real, pretend they were the child in the movie, and react as if they were in that situation. Prior to each video clip, participants were given instructions for one of four conditions: (1) focused attention to breath, BREATH, (2) mindful acceptance, ACCEPT, (3) distraction, DISTRACT, or (4) passive viewing, LOOK (Supplemental Methods 1.2). Participants were cued using the text and visual cue during the task in the fMRI scanner. Each trial lasted $30 \mathrm{~s}$, and included a $2 \mathrm{~s}$ instruction slide, a $30 \mathrm{~s}$ video clip, and a $5 \mathrm{~s}$ emotion rating period (Figure 1 ). The inter-trial interval $(2 \mathrm{~s})$ was a "RELAX" screen. There were 5 trials of each of the 4 conditions, for a total of 20 trials (total time $=13 \mathrm{~min}, 21 \mathrm{sec}$ ). Video clips were counterbalanced across conditions. Presentation software (Neurobehavioral Systems, Inc.) was used for stimulus presentation and behavioral data acquisition. The task was displayed on a back-projection

screen affixed to the head coil and behavioral responses were registered using a $2 \mathrm{x} 2 \mathrm{MR}$-compatible response 
device.

\subsection{Emotional distress ratings}

Distress ratings were averaged for each condition (BREATH, ACCEPT, DISTRACT, LOOK) and submitted to IBM SPSS software v.26 for within-subject analysis. Overall effects of condition were examined using a one-way nonparametric Friedman test for repeated-measures $(p<0.05)$. Post hoc repeated-measures Wilcoxon Signed-Ranks tests were used to further examine differences in distress ratings between conditions. Behavioral data were missing for one subject due to errors in data collection.

2.5 fMRI data acquisition and analysis

Details regarding blood-oxygen-level-dependent (BOLD) imaging fMRI data acquisition, preprocessing, quality assurance, and first-level analyses are provided in the Supplemental Methods.

2.5.1 Second-level analysis: Group-level random effects analysis was performed in SPM8 to examine withinsubjects differential activation patterns based on instruction, using one-sample $t$-tests. First, to identify regions modulated by meditation emotion regulation techniques vs. a control condition, we examined the contrasts BREATH vs. LOOK and ACCEPT vs. LOOK. To identify regions modulated by the nonmeditation emotion regulation condition, we examined DISTRACT vs. LOOK. Then, we compared the two meditation conditions (BREATH vs. ACCEPT) and compared the meditation vs. non-meditation instructions (i.e., BREATH vs. DISTRACT, ACCEPT vs. DISTRACT).

2.6.2 DMN regions of interest (ROI) analyses: To identify the effects of different emotion regulation techniques on brain activity, we focused on a priori ROIs in the DMN: MFC, posterior cingulate cortex, dorsal frontal cortex, and supplementary motor area. Small-volume familywise error (FWE) correction was used to identify significant ROI results ( $p F W E$ [?] 0.05 and 5-voxel minimum).

2.6.3 Whole brain analyses: A complementary whole-brain analysis was performed using a whole-brain FWE corrected threshold $(p F W E<0.05)$. See supporting Information for details.

\section{Results}

\subsection{Distress ratings}

There was no main effect of condition (BREATH, ACCEPT, DISTRACT, LOOK) on distress ratings following negative video clips, Friedman's two-way analysis of variance by ranks, $\mathrm{df}=3$, test stat $=2.35, p=0.5$ (Supplemental Material Table S1). Post hoc repeated measures Wilcoxon Signed-Ranks tests also revealed no significant differences between conditions, $p^{\prime} \mathrm{s}>0.1$.

\section{2 fMRI results}

\subsubsection{Effects of meditation emotion regulation strategies (vs. passive viewing)}

A whole-brain BREATH vs. LOOK contrast revealed lower activity in several regions of the DMN, including the precuneus, parahippocampal gyrus, and posterior cingulate cortex, and also the cuneus and lingual gyrus (Table 2, Figure 2b ). There were no clusters showing the opposite pattern (i.e., higher activity for BREATH relative to LOOK; Table 2 ) and no significant clusters for the BREATH vs. LOOK contrast in any DMN ROI (pFWE >0.05). There were also no significant clusters for the ACCEPT vs. LOOK contrast at the whole-brain threshold (Table 2 ) or in any DMN ROI $(p F W E>0.05)$.

\subsubsection{Effects of a non-meditation emotion regulation strategy (vs. passive viewing)}

There were no significant clusters for the DISTRACT vs. LOOK contrast at the whole-brain threshold (Table 2 ) or in any DMN ROI $(p F W E>0.05)$.

\subsubsection{Comparison between meditation emotion regulation strategies}

ROI analyses showed differential DMN activity for the BREATH vs. ACCEPT contrast in the MFC, such that MFC activation was lower during the BREATH relative to the ACCEPT condition $[\mathrm{xyz}=0,58,18,9$ 
voxels, $Z=3.73, p F W E=0.02] \mathbf{2 a}$. No other ROIs were significant for the BREATH vs. ACCEPT contrast and there were no significant results for this contrast at the whole-brain corrected threshold (Table 3 ). 3.2.4 Comparison between meditation and non-meditation emotion regulation strategies

A whole-brain BREATH vs. DISTRACT contrast revealed lower activation in the precuneus and middle temporal gyrus at the whole-brain corrected threshold (Figure 2a, Table 3 ). No ROIs showed significant activation for the BREATH vs. DISTRACT contrast. For the ACCEPT vs. DISTRACT contrast, there was lower DMN activation in the MFC ROI $[\mathrm{xyz}=2,48,22,6$ voxels, $Z=3.5, p F W E=0.05]$, Figure 2b. No other ROIs were significant for the ACCEPT vs. DISTRACT contrast and there were no significant clusters for this contrast at the whole-brain threshold (Table 3 ).

\subsection{Exploratory analyses}

\subsubsection{Associations between brain activation and distress ratings}

Distress ratings were not significantly associated with activation in any brain region that showed significant results in whole-brain or ROI analyses ( $p$ 's $>0.05)$.

\subsubsection{Associations between distress ratings and demographic or clinical measures}

Distress ratings were not associated with age, anxiety symptoms, or trait mindfulness ( $p$ 's $>0.05)$. However, Spearman correlation indicated that older age at diagnosis was associated with lower self-reported distress following the DISTRACT condition, $r(10)=-0.75, p=0.013$.

\subsubsection{Associations between brain activation and demographic or clinical measures}

Distress ratings were not associated with age at diagnosis, anxiety symptoms, or trait mindfulness ( $p$ 's $>$ $0.05)$. However, older children demonstrated lower brain activation in the posterior cingulate cortex $[\mathrm{xyz}=$ $-22,-68,8]$ during the BREATH vs. LOOK contrast as compared to younger children, $r(12)=-0.58, p=$ 0.049 . Older children also displayed lower activation in the middle temporal gyrus during the BREATH vs. DISTRACT contrast than younger children, $r(12)=-0.62, p=0.032$.

\section{Discussion}

This is the first study, to our knowledge, to investigate the neural correlates of active meditation as an emotion regulation technique in a pediatric sample. We examined neural activation in children with cancer while they viewed distress-inducing video clips and engaged in meditation (focused attention, mindful acceptance) or non-meditation (distraction) emotion regulation techniques. In the control condition, participants passively viewed the video clip. The following findings emerged: (1) compared to the control condition, mediation emotion regulation techniques were associated with lower activity in several regions of the DMN. (2) The non-meditation condition - distraction - was not associated with a similar reduction in neural activation as compared to the control condition, suggesting that meditation emotion regulation techniques may be more effective for downregulating DMN activity. (3) meditation emotion regulation strategies were associated with lower DMN activity than distraction. Distraction is often used to manage children's pain in clinical settings, (e.g., needle-related procedures). ${ }^{48}$ Although there were no differences in distress ratings between conditions, evidence that meditation emotion regulation is more effective at quelling DMN activity may suggest longerterm benefits - a hypothesis that requires future study with larger sample sizes. These findings may have implications for understanding the neural mechanisms underlying meditation-based emotion regulation in children.

Both forms of meditation emotion regulation strategies examined in this study were associated with lower activity in DMN regions, including the parahippocampal gyrus, precuneus, and posterior cingulate cortex. Lower activity in the DMN during active meditation is consistent with prior fMRI studies in adults. For example, a meta-analysis of 78 fMRI studies in adults revealed consistent de-activations in DMN regions (e.g., posterior cingulate cortex) during focused attention meditation. ${ }^{37}$ Another fMRI study in meditation-naïve adults reported that mindful acceptance emotion regulation reduced pain-related activation in the DMN (e.g., posterior cingulate, precuneus). ${ }^{16}$ This suggests that children with minimal training may benefit from 
meditation. We did not detect activation in the CEN during meditation, consistent with studies showing that meditation does not engage CEN control systems ${ }^{49}$. We also did not observe CEN activation for distraction, which may reflect underdevelopment of cognitive forms of emotion regulation and the $\mathrm{CEN}^{30,31}$.

Our whole-brain and ROI analyses revealed lower DMN activation for meditation emotion regulation techniques, but not for distraction. Prior studies on cognitive forms of emotion regulation (e.g., distraction) do not consistently report activation changes in the $\mathrm{DMN}^{49,50}$ and there is evidence that DMN engagement changes across development ${ }^{30}$. Distraction is considered an attentional deployment strategy that re-directs attention away from emotion-eliciting stimuli ${ }^{51}$ and engages the CEN in adults. ${ }^{49}$ Here, both meditation techniques (focused attention, mindful acceptance) were associated with lower DMN activity than distraction. Specifically, focused attention was associated with lower activation in the precuneus, a DMN region associated with maintaining self-consciousness during self-referential processes. ${ }^{52}$ Lower activity in the precuneus during focused attention is consistent with meta-analytic findings in adults. ${ }^{37}$ Therefore, focused attention meditation may suppress self-referential thoughts evoked by negative stimuli and protect against depressive rumination in children. Relative to distraction, mindful acceptance was associated with lower activity in the MFC, consistent with a prior emotion regulation study in adults. ${ }^{16}$ Other studies have reported reduced activation in the MFC during sustained meditation, ${ }^{53}$ negative autobiographical memory recall, and acceptance of present emotional state. ${ }^{54}$ Taken together, lower MFC activation observed during mindful acceptance may reflect reduced elaboration and appraisal of emotional experiences. Although distraction and meditation techniques are effective for reducing children's pain and distress, the present findings suggest that meditation techniques may be more effective for modulating DMN activity in children. Given that higher DMN activity is implicated in depressive rumination, ${ }^{28,54}$ these findings imply a potential long-term protective role of meditation for helping children cope with stressful experiences.

Although we observed differences in the brain between conditions, there were no differences in distress ratings. We may have been underpowered to detect changes in distress ratings. Prior studies support beneficial effects of meditation on self-reported pain and emotional distress in children over time (e.g., over 4- or 8 -week programs ${ }^{32}$ or following a single session. ${ }^{21}$ We also observed minimal differences in brain activity between conditions, which should be examined in future studies with larger sample sizes. However, we found preliminary evidence that focused attention may be more effective for downregulating DMN activity than mindful acceptance, which may reflect the fact that paying attention to the breath may be a simpler concept for children. This finding is interesting given results of a prior study in healthy adults showing that experienced meditators were better than beginners at downregulating MFC activity in response to emotional images. ${ }^{17}$ Therefore, more experience with meditation may improve attenuation of DMN activation.

\subsection{Limitations}

Our sample consisted of a relatively small sample size of children with cancer and results may reflect the unique neurobiology of this population. We used a within-subjects design and multi-echo fMRI imaging techniques to increase study power. Sampling limitations are attributed to the low base rate of childhood cancer, which precluded our ability to examine sex differences in neural or behavioral measures. However, the sample size in the present study $(n=12)$ is consistent with previously published neuroimaging studies in pediatric cancer survivors (e.g., $n=8,15) .{ }^{55,56}$ We focused on children with cancer because this pediatric population is exposed to disease- and treatment-related stress, is at increased risk of emotion dysregulation, ${ }^{35}$ and because martial arts-based meditation programs have shown to be effective for reducing pain and emotional distress in this population. ${ }^{21,36}$ In addition, the relatively wide age span (5-17 years) is a limitation due to differences in functional connectivity in young children compared to older teenagers. Another limitation is that we examined two forms of meditation (i.e., focused attention, mindful acceptance) and one cognitive emotion regulation strategy (distraction), which may not be representative of all available emotion regulation techniques. In addition, we did not have a non-distressing (e.g., positively-valenced) condition to compare our activation patterns to, so it is unclear if the resulting neural activation patterns are specific to regulation of negative stimuli. Additionally, all participants had at least four hours of meditation instruction. Findings may differ based on meditation experience, ${ }^{17}$ as these techniques become more automatized. Therefore, future 
studies should examine different forms of meditation and the impact of meditation experience.

\subsection{Conclusions}

Mounting evidence indicates that meditation is an effective approach for regulating pediatric pain and emotional distress. Mindfulness- and meditation-based interventions may be particularly well-suited for children because they do not engage CEN control systems, which are underdeveloped in youth, are easily accessible to children, and can be adapted to developmental age. Indeed, the present findings suggest that simple forms of meditation, such as focused attention to breath and mindful acceptance, can reduce activity in the DMN in children with limited meditation experience. Given alterations in the DMN are linked to various forms of psychopathology, ${ }^{57}$ these results suggest that meditation emotion regulation techniques may be effective for modulating DMN activity in youth. These results also have implications for the management of pain and distress in pediatric healthcare settings (e.g., needle-related procedures), and for mitigating negative effects of stress and trauma in pediatric population that face disproportionally high stress (e.g., chronic health conditions).

\section{Acknowledgements}

The authors thank Richard Plowden, Michael Hunt, and Peter Davenport of Kids Kicking Cancer for facilitating the martial arts training; Autumm Heeter, Shelley Paulisin, Xhenis Brahimi, Craig Peters, and Farrah Elrahal for assistance with data collection and analysis and study management; Kristopher Dulay and Wendy Henning of Children's Hospital of Michigan for assistance with participant recruitment; Pavan Jella and Dr. Richard Genik at the WSU MR Research Facility with assistance with imaging data acquisition

\section{Conflict of interest}

EG is founder and global director and MB is the scientific director of Kids Kicking Cancer, a non-profit organization that developed the martial arts intervention. This work was supported, in part, by the St. Baldrick's Foundation and the National Institute of Mental Health to HM, and HM has received previous grant funding from Kids Kicking Cancer. The authors declare no other conflicts of interest.

\section{Funding}

This work was supported, in part, by the St. Baldrick's Foundation [SBF523497 to H.M.]; and from the National Institute of Mental Health [K01MH119241 to H.M]. Funders were not involved in the conduct of the study, data analysis or interpretation, or decision to publish.

\section{References}

1. Martin RE, Ochsner KN. The neuroscience of emotion regulation development: Implications for education. Curr Opin Behav Sci . 2016. doi:10.1016/j.cobeha.2016.06.006

2. Graziano PA, Reavis RD, Keane SP, Calkins SD. The role of emotion regulation in children's early academic success. J Sch Psychol . 2007. doi:10.1016/j.jsp.2006.09.002

3. Rydell AM, Thorell LB, Bohlin G. Emotion regulation in relation to social functioning: An investigation of child self-reports. Eur J Dev Psychol . 2007. doi:10.1080/17405620600783526

4. Aldao A, Nolen-Hoeksema S, Schweizer S. Emotion-regulation strategies across psychopathology: A metaanalytic review. Clin Psychol Rev . 2010. doi:10.1016/j.cpr.2009.11.004

5. Althoff RR, Verhulst FC, Rettew DC, Hudziak JJ, Van Der Ende J. Adult outcomes of childhood dysregulation: A 14-year follow-up study. J Am Acad Child Adolesc Psychiatry . 2010. doi:10.1016/j.jaac.2010.08.006

6. Pandey A, Hale D, Das S, Goddings AL, Blakemore SJ, Viner RM. Effectiveness of universal self-regulationbased interventions in children and adolescents a systematic review and meta-analysis.JAMA Pediatr . 2018. doi:10.1001/jamapediatrics.2018.0232

7. Hanh TN. The Miracle of Mindfulness! : A Manual of Meditation .; 1976. 
8. Lutz A, Slagter HA, Dunne JD, Davidson RJ. Attention regulation and monitoring in meditation. Trends Cogn Sci . 2008. doi:10.1016/j.tics.2008.01.005

9. Tang YY, Holzel BK, Posner MI. The neuroscience of mindfulness meditation. Nat Rev Neurosci . 2015. doi:10.1038/nrn3916

10. Bishop SR, Lau M, Shapiro S, et al. Mindfulness: A proposed operational definition. Clin Psychol Sci Pract . 2004. doi:10.1093/clipsy/bph077

11. Tang YY, Holzel BK, Posner MI. Traits and states in mindfulness meditation. Nat Rev Neurosci . 2016. doi:10.1038/nrn.2015.7

12. Baer RA. Mindfulness training as a clinical intervention: A conceptual and empirical review. Clin Psychol Sci Pract . 2003. doi:10.1093/clipsy/bpg015

13. Goyal M, Singh S, Sibinga E, et al. Meditation programs for psychological stress and well-being: A systematic review and meta-analysis. JAMA Intern Med . 2014;174(3):357-368. doi:10.1001/jamainternmed.2013.13018

14. McClintock AS, Rodriguez MA, Zerubavel N. The Effects of Mindfulness Retreats on the Psychological Health of Non-clinical Adults: a Meta-analysis. Mindfulness (N Y) . 2019. doi:10.1007/s12671-019-01123-9

15. Xunlin N, Lau Y, Klainin-Yobas P. The effectiveness of mindfulness-based interventions among cancer patients and survivors: a systematic review and meta-analysis. Support Care Cancer . 2020. doi:10.1007/s00520-019-05219-9

16. Kober H, Buhle J, Weber J, Ochsner KN, Wager TD. Let it be: Mindful acceptance down-regulates pain and negative emotion. Soc Cogn Affect Neurosci . 2019;14(11):1147-1158. doi:10.1093/scan/nsz104

17. Taylor VA, Grant J, Daneault V, et al. Impact of mindfulness on the neural responses to emotional pictures in experienced and beginner meditators. Neuroimage . 2011. doi:10.1016/j.neuroimage.2011.06.001

18. Dunning DL, Griffiths K, Kuyken W, et al. Research Review: The effects of mindfulness-based interventions on cognition and mental health in children and adolescents - a meta-analysis of randomized controlled trials. J Child Psychol Psychiatry Allied Discip . 2019. doi:10.1111/jcpp.12980

19. Semple RJ, Droutman V, Reid BA. MINDFULNESS GOES TO SCHOOL: THINGS LEARNED (SO FAR) FROM RESEARCH AND REAL-WORLD EXPERIENCES. Psychol Sch . 2017. doi:10.1002/pits.21981

20. Bluth M, Thomas R, Cohen C, Bluth A, Goldberg RE. Martial arts intervention decreases pain scores in children with malignancy.Pediatr Heal Med Ther . 2016; Volume 7:79-87. doi:10.2147/phmt.s104021

21. Marusak HA, Cohen C, Goldberg E, et al. Martial Arts-Based Therapy Reduces Pain and Distress Among Children with Chronic Health Conditions and Their Siblings. 2020.

22. Ortiz R, Sibinga E. The Role of Mindfulness in Reducing the Adverse Effects of Childhood Stress and Trauma. Children . 2017. doi:10.3390/children4030016

23. Kessler RC, Amminger GP, Aguilar-Gaxiola S, Alonso J, Lee S, Ustun TB. Age of onset of mental disorders: A review of recent literature.Curr Opin Psychiatry . 2007. doi:10.1097/YCO.0b013e32816ebc8c

24. Ochsner KN, Silvers JA, Buhle JT. Review and evolving model of the cognitive control of emotion. Ann N Y Acad Sci . 2012;(1251):E1-E24. doi:10.1111/j.1749-6632.2012.06751.x.Functional

25. Greicius MD, Krasnow B, Reiss AL, Menon V. Functional connectivity in the resting brain: A network analysis of the default mode hypothesis.Proc Natl Acad Sci U S A . 2003. doi:10.1073/pnas.0135058100

26. Farb NAS, Segal Z V., Mayberg H, et al. Attending to the present: Mindfulness meditation reveals distinct neural modes of self-reference.Soc Cogn Affect Neurosci . 2007. doi:10.1093/scan/nsm030 
27. Fox KCR, Spreng RN, Ellamil M, Andrews-Hanna JR, Christoff K. The wandering brain: Meta-analysis of functional neuroimaging studies of mind-wandering and related spontaneous thought processes.Neuroimage . 2015. doi:10.1016/j.neuroimage.2015.02.039

28. Zhou HX, Chen X, Shen YQ, et al. Rumination and the default mode network: Meta-analysis of brain imaging studies and implications for depression. Neuroimage . 2020. doi:10.1016/j.neuroimage.2019.116287

29. Sheline YI, Barch DM, Price JL, et al. The default mode network and self-referential processes in depression. Proc Natl Acad Sci U S A . 2009. doi:10.1073/pnas.0812686106

30. McRae K, Gross JJ, Weber J, et al. The development of emotion regulation: An fMRI study of cognitive reappraisal in children, adolescents and young adults. Soc Cogn Affect Neurosci . 2012. doi:10.1093/scan/nsr093

31. Qin S, Young CB, Supekar K, Uddin LQ, Menon V. Immature integration and segregation of emotionrelated brain circuitry in young children.Proc Natl Acad Sci U S A . 2012. doi:10.1073/pnas.1120408109

32. Bauer CCC, Caballero C, Scherer E, et al. Mindfulness training reduces stress and Amygdala reactivity to fearful faces in middle-school children. Behav Neurosci . 2019;133(6):569-585. doi:10.1037/bne0000337

33. Bauer CCC, Rozenkrantz L, Caballero C, et al. Mindfulness training preserves sustained attention and resting state anticorrelation between default-mode network and dorsolateral prefrontal cortex: A randomized controlled trial. Hum Brain Mapp . 2020. doi:10.1002/hbm.25197

34. Marusak HA, Elrahal F, Peters CA, et al. Mindfulness and dynamic functional neural connectivity in children and adolescents. Behav Brain Res . 2018;336:211-218. doi:10.1016/j.bbr.2017.09.010

35. Marusak HA, Harper FW, Taub JW, Rabinak CA. Pediatric cancer, posttraumatic stress and fear-related neural circuitry. Int J Hematol Oncol . 2019;8(2):IJH17. doi:10.2217/ijh-2019-0002

36. Bluth M, Thomas R, Cohen C, Bluth A, Goldberg RE. Martial arts intervention decreases pain scores in children with malignancy.Pediatr Heal Med Ther . 2016;Volume 7:79-87. doi:10.2147/PHMT.S104021

37. Fox KCR, Dixon ML, Nijeboer S, et al. Functional neuroanatomy of meditation: A review and meta-analysis of 78 functional neuroimaging investigations. Neurosci Biobehav Rev . 2016;65:208-228. doi:10.1016/j.neubiorev.2016.03.021

38. Birmaher B, Khetarpal S, Brent D, et al. The Screen for Child Anxiety Related Emotional Disorders (SCARED): Scale construction and psychometric characteristics. J Am Acad Child Adolesc Psychiatry . 1997. doi:10.1097/00004583-199704000-00018

39. Birmaher B, Brent D, Chiappetta L, Bridge J, Monga S, Baugher M. Psychometric properties of the Screen for Child Anxiety Related Emotional Disorders (SCARED): a replication study. J Am Acad Child Adolesc Psychiatry . 1999;38(10):1230-1236.

40. Desousa DA, Salum GA, Isolan LR, Manfro GG. Sensitivity and specificity of the Screen for Child Anxiety Related Emotional Disorders (SCARED): A community-based study. Child Psychiatry Hum Dev . 2013;44(3):391-399. doi:10.1007/s10578-012-0333-y

41. Greco L a., Baer, R. A. \&, Smith GT. Child and Adolescent Mindfulness Measure (CAMM). Psychol Assess . 2011;23(3):606-614. doi:10.1037/a0022819

42. Prenoveau JM, Papadakis AA, Schmitz JCS, Hirsch EL, Dariotis JK, Mendelson T. Psychometric properties of the child and adolescent mindfulness measure (CAMM) in racial minority adolescents from low-income environments. Psychol Assess . 2018. doi:10.1037/pas0000630

43. Dickenson J, Berkman ET, Arch J, Lieberman MD. Neural correlates of focused attention during a brief mindfulness induction. Soc Cogn Affect Neurosci . 2013;8(1):40-47. doi:10.1093/scan/nss030 
44. Goldin P, Gross J. Effects of mindfulness-based stress reduction (MBSR) on emotion regulation in social anxiety disorder. Emotion . 2010;10(1):83-91. doi:10.1037/a0018441

45. Trentacosta CJ, Harper FWK, Albrecht TL, Taub JW, Phipps S, Penner LA. Pediatric Cancer Patients' Treatment-Related Distress and Longer-Term Anxiety: An Individual Differences Perspective. J Dev Behav Pediatr . 2016;37(9):753-761. doi:10.1097/DBP.0000000000000327

46. Wong D, Baker C. Pain in Children: Comparison of Assessment Scales.Pediatr Nurs . 1988;14(1):9-17.

47. Zimmer-Gembeck MJ, Lees DC, Bradley GL, Skinner EA. Use of an analogue method to examine children's appraisals of threat and emotion in response to stressful events. Motiv Emot . 2009;33(2):136-149. doi:10.1007/s11031-009-9123-7

48. Birnie KA, Noel M, Chambers CT, Uman LS, Parker JA. Psychological interventions for needlerelated procedural pain and distress in children and adolescents. Cochrane Database Syst Rev . 2018. doi:10.1002/14651858.CD005179.pub4

49. Moodie CA, Suri G, Goerlitz DS, et al. The neural bases of cognitive emotion regulation: The roles of strategy and intensity. Cogn Affect Behav Neurosci . 2020. doi:10.3758/s13415-020-00775-8

50. Buhle JT, Silvers JA, Wage TD, et al. Cognitive reappraisal of emotion: A meta-analysis of human neuroimaging studies. Cereb Cortex . 2014. doi:10.1093/cercor/bht154

51. Gross JJ. The emerging field of emotion regulation: An integrative review. Rev Gen Psychol . 1998. doi:10.1037/1089-2680.2.3.271

52. Fransson P, Marrelec G. The precuneus/posterior cingulate cortex plays a pivotal role in the default mode network: Evidence from a partial correlation network analysis. Neuroimage . 2008. doi:10.1016/j.neuroimage.2008.05.059

53. Baerentsen KB, Stodkilde-Jorgensen H, Sommerlund B, et al. An investigation of brain processes supporting meditation. Cogn Process . 2010. doi:10.1007/s10339-009-0342-3

54. Kross E, Davidson M, Weber J, Ochsner K. Coping with Emotions Past: The Neural Bases of Regulating Affect Associated with Negative Autobiographical Memories. Biol Psychiatry . 2009. doi:10.1016/j.biopsych.2008.10.019

55. Kesler SR, Gugel M, Pritchard-Berman M, et al. Altered resting state functional connectivity in young survivors of acute lymphoblastic leukemia. Pediatr Blood Cancer . 2014;61(7):1295-1299. doi:10.1002/pbc. 25022

56. Robinson KE, Livesay KL, Campbell LK, et al. Working memory in survivors of childhood acute lymphocytic leukemia: functional neuroimaging analyses. Pediatr Blood Cancer . 2010;54(4):585-590. doi: $10.1002 /$ pbc. 22362

57. Broyd SJ, Demanuele C, Debener S, Helps SK, James CJ, Sonuga-Barke EJS. Default-mode brain dysfunction in mental disorders: A systematic review. Neurosci Biobehav Rev . 2009. doi:10.1016/j.neubiorev.2008.09.002

\section{Tables}

TABLE 1 Participant demographics and clinical characteristics

\begin{tabular}{llll}
\hline Variable & $\mathrm{n}(\%)$ & $M(S D)$ & Range \\
\hline Age (yrs) & & $10.33(3.26)$ & $5-17$ \\
Age at diagnosis (years) & $5(42 \%)$ & $5.48(4.49)$ & $1-17$ \\
$\begin{array}{l}\text { Biological sex (females) } \\
\text { Length of treatment (years) }\end{array}$ & & $3.06(0.73)$ & $2-4$
\end{tabular}




\begin{tabular}{llll}
\hline Variable & $\mathrm{n}(\%)$ & $M(S D)$ & Range \\
\hline Cancer diagnosis & Cancer diagnosis & Cancer diagnosis & Cancer diagnosis \\
Acute lymphoblastic leukemia (ALL) & $8(67 \%)$ & & \\
Acute promyelocytic leukemia (APML) & $1(8 \%)$ & & \\
Neuroblastoma & $1(8 \%)$ & & \\
Wilms Tumor & $1(8 \%)$ & & \\
Juvenile myelomonocytic leukemia & $1(8 \%)$ & & \\
Race/ethnicity & & & \\
Caucasian, Non-Hispanic & $7(58.3 \%)$ & & \\
African American, Non-Hispanic & $3(25 . \%)$ & & \\
Other & $1(8.3 \%)$ & & \\
Not reported & $1(8.3 \%)$ & & \\
Annual household income & & & \\
$\$ 0-10000$ & $1(8.3 \%)$ & & \\
$\$ 10$ 000-20 000 & $1(8.3 \%)$ & & \\
$\$ 20$ 000-30 000 & $2(16.7 \%)$ & & \\
$\$ 30$ 000-40 000 & $1(8.3 \%)$ & & \\
$\$ 40$ 000-50 000 & $1(8.3 \%)$ & & \\
$\$ 50$ 000-60 000 & $0(0 \%)$ & & \\
$\$ 60$ 000-80 000 & $3(25 \%)$ & & \\
$\$ 80$ 000-100 000 & $1(8.3 \%)$ & & \\
$\$ 100$ 000-120 000 & $0(0 \%)$ & & \\
$\$ 120$ 000-140 000 & $1(8.3 \%)$ & & \\
Not reported & $1(8.3 \%)$ & & \\
Trait mindfulness (CAMM) & & & \\
Anxiety symptoms (SCARED) & & & \\
\hline & & & \\
\end{tabular}

SCARED — Screen for Child Anxiety-Related Emotional Disorders; CAMM — Child and Adolescent Mindfulness Measure.

TABLE 2 Whole brain results for regions showing differential activation while participants are engaged in meditation and non-meditation emotion regulation techniques vs. the control condition.

\begin{tabular}{ll}
\hline Regions of activation & BA \\
\hline Meditation techniques (ACCEPT, BREATH) vs. the control condition (LOOK) & Meditation techniques (ACCEPT, BREA \\
$L O O K>B R E A T H$ & $L O O K>B R E A T H$ \\
Cuneus & 17 \\
Posterior cingulate cortex & 30 \\
Posterior cingulate cortex & 30 \\
Posterior cingulate cortex & 30 \\
Parahippocampal gyrus & 30 \\
Posterior cingulate cortex & 30 \\
Lingual gyrus & 18 \\
Precuneus & 31 \\
$L O O K<B R E A T H$ & $L O O K<B R E A T H$ \\
No significant clusters & No significant clusters \\
$L O O K>A C C E P T$ & $L O O K>A C C E P T$ \\
No significant clusters & No significant clusters \\
$L O O K<A C C E P T$ & $L O O K<A C C E P T$
\end{tabular}




\begin{tabular}{ll}
\hline Regions of activation & BA \\
\hline No significant clusters & No significant clusters \\
Non-meditation technique (DISTRACT) vs. the control condition (LOOK) & Non-meditation technique (DISTRACT) \\
LOOK $>$ DISTRACT & LOOK $>$ DISTRACT \\
No significant clusters & No significant clusters \\
$L O O K<D I S T R A C T$ & $L O O K<D I S T R A C T$ \\
No significant clusters & No significant clusters \\
\hline
\end{tabular}

BA — Brodmann Area; N/A — Not applicable; BOLD — Blood-oxygen level-dependent; BREATH — focus attention to the breath; ACCEPT — pay attention to emotions in a non-judgmental way; DISTRACT count backwards from ten; LOOK - passively view the movie. X, y, and $\mathrm{z}$ are MNI peak coordinates. Voxel extent refers to the spatial extent of each cluster. R/L refers to lateralization of activation. Results are corrected at the whole-brain level (voxelwise $p<0.001$; cluster extent $=6$ voxels).

TABLE 3 Whole brain results for regions showing differential activation during different types of emotion regulation techniques (i.e., BREATH vs. ACCEPT vs. DISTRACT).

Regions of activation

Comparison between meditation techniques (BREATH vs. ACCEPT)

BREATH>ACCEPT: No significant clusters

BREATH $<$ ACCEPT: No significant clusters

Comparison between meditation and non-meditation techniques (BREATH vs. DISTRACT, ACCEPT vs. DISTRACT)

BREATH>DISTRACT: No significant clusters

BREATH $<$ DISTRACT

Middle temporal gyrus

Precuneus

ACCEPT $>$ DISTRACT: No significant clusters

ACCEPT $<$ DISTRACT: No significant clusters

BA - Brodmann Area; N/A — Not applicable; BOLD - Blood-oxygen level-dependent; BREATH — focus attention to the breath; ACCEPT - pay attention to emotions in a non-judgmental way; DISTRACT count backwards from ten; LOOK - passively view the movie. X, y, and $\mathrm{z}$ are MNI peak coordinates. Voxel extent refers to the spatial extent of each cluster. R/L refers to lateralization of activation. Results are corrected at the whole-brain level (voxelwise $p<0.001$; cluster extent $=6$ voxels).

Figures 


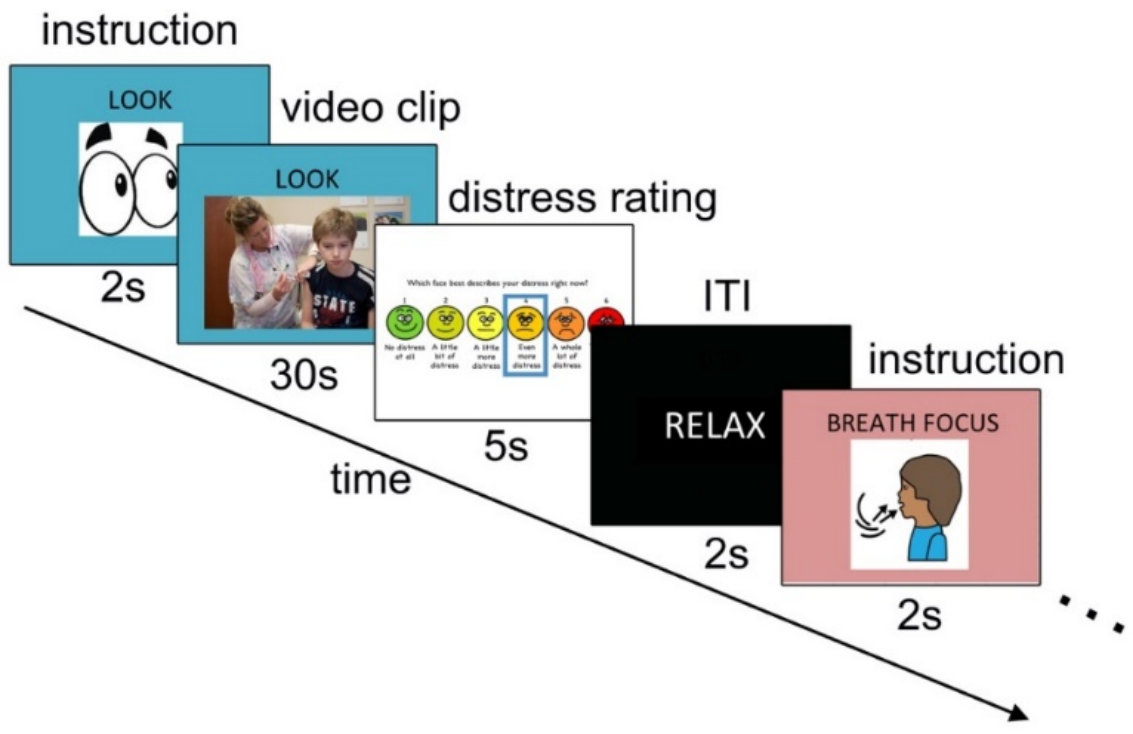

FIGURE 1 Emotion regulation task. Participants received one of four instructions prior to watching the video clip. After each clip, participants rated their level of distress $(1=$ no distress at all, $6=$ the worst possible distress) on a visual analog scale (VAS), which is an adapted version of the Wong and Baker's FACES scale. Instructions consisted of (1) LOOK, a control condition during which participants were instructed to passively view the video clip, (2) DISTRACT, a non-meditation condition during which participants were instructed to count backwards from ten, and two meditation emotion regulation techniques: (3) BREATH, during which participants were instructed to focus their attention on their breathing, and (4) ACCEPT, during which participants were instructed to pay attention to the emotions they were experiencing in a nonjudgement way. 


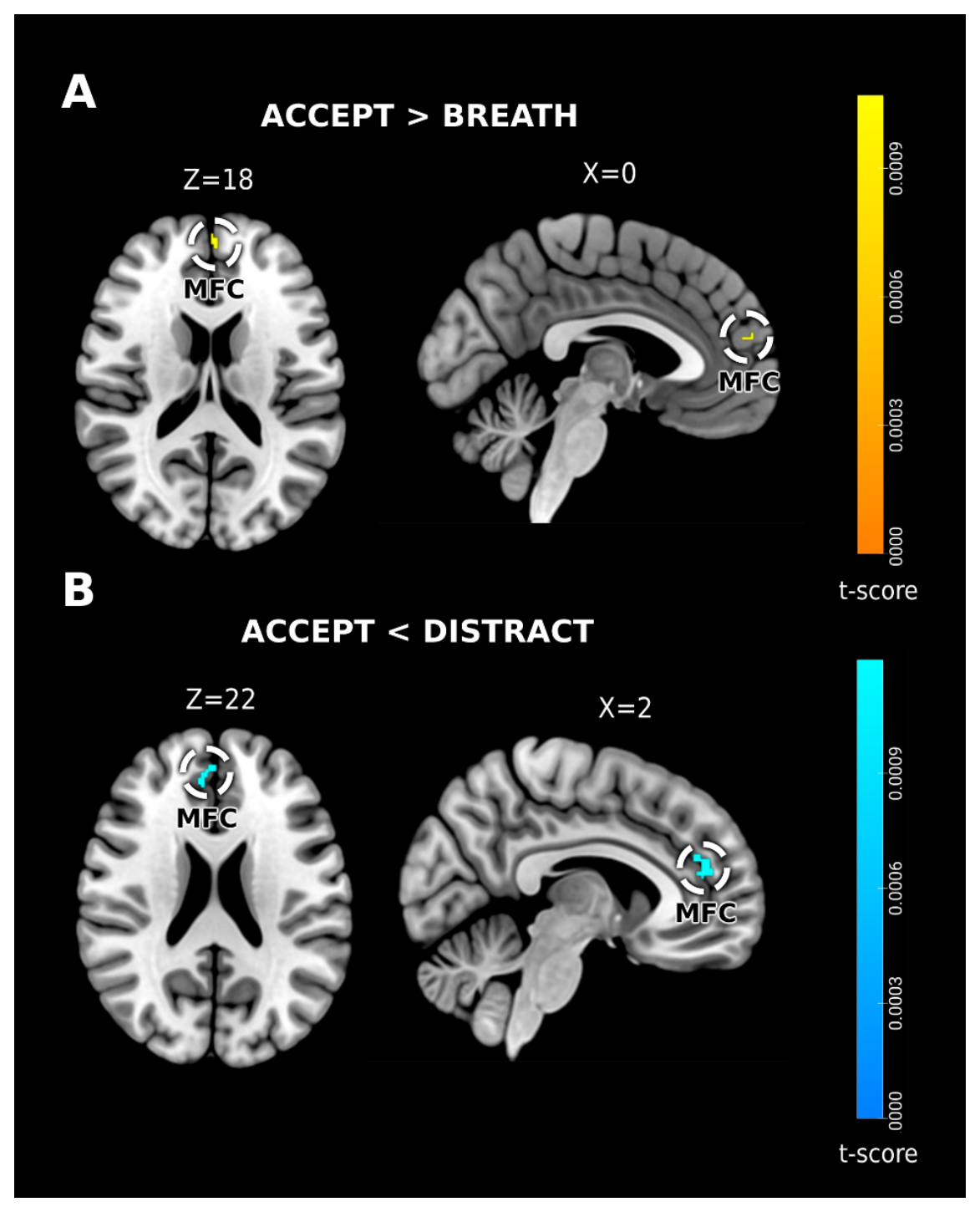

FIGURE 2 Region of interest results: Effects of meditation emotion regulation techniques on activation in the default mode network (DMN). (a) Differential DMN activation associated with ACCEPT vs. BREATH meditation instructions. Yellow colors indicate greater BOLD response in the DMN for ACCEPT vs. BREATH. (b) Differential DMN activation during meditation instructions (ACCEPT) vs. a non- meditation instruction (DISTRACT). Blue colors indicate lower DMN activation during meditation instructions (ACCEPT) relative to non- meditation instructions (DISTRACT). Results significant at small-volume corrected threshold ( $p F W E$ [?] $0.05,>5$ voxels) and shown at $p<0.005$ for display purposes. BOLD - Blood-oxygen level-dependent; MFC - medial frontal cortex. X, y, and z are MNI peak coordinates. 


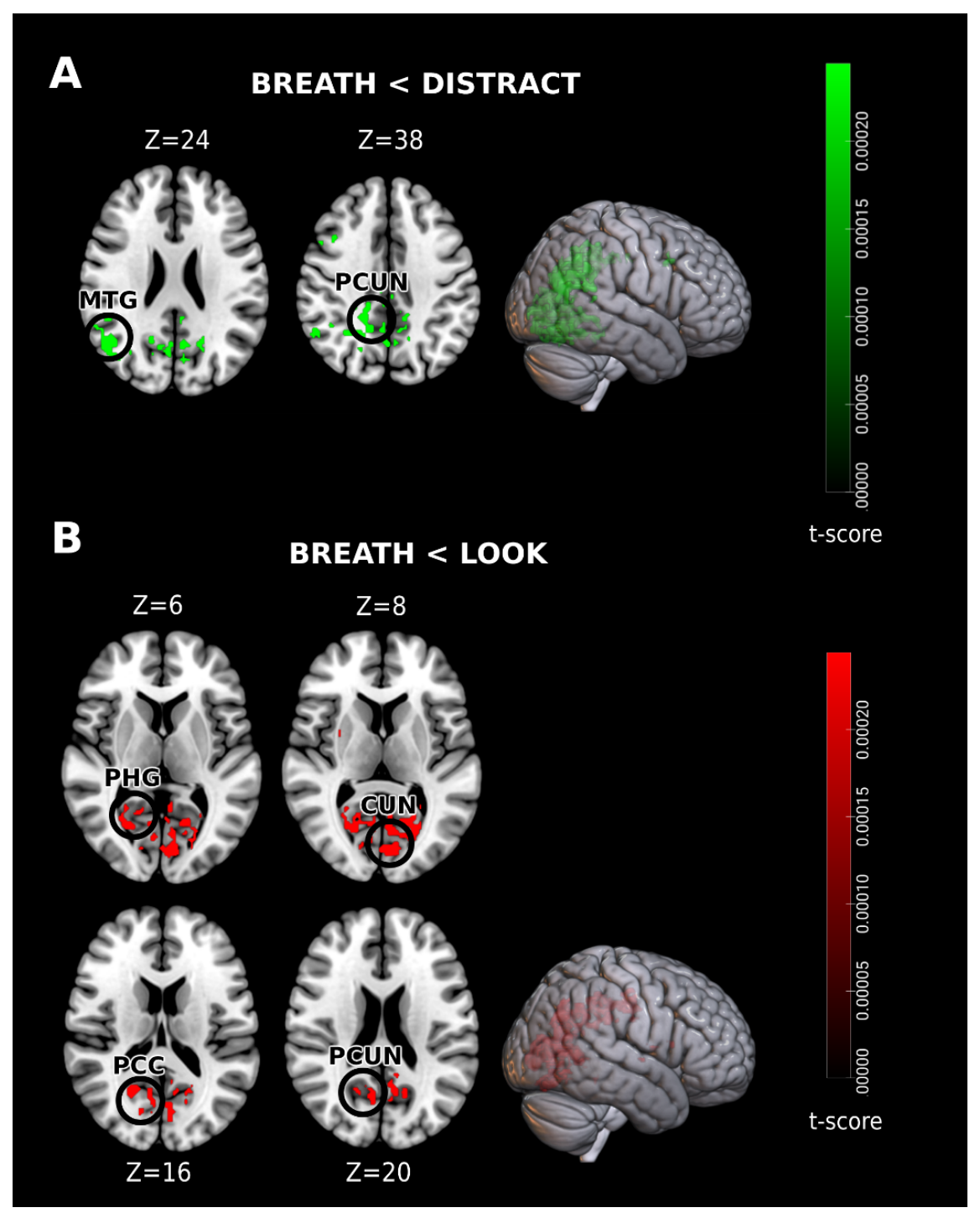

FIGURE 3 Whole brain results: Effects of meditation emotion regulation techniques on activation in the default mode network (DMN). (a) Differential DMN activation associated with meditation (BREATH) vs. non-meditation (DISTRACT) instructions. Green colors indicate lower BOLD response in the DMN for BREATH vs. DISTRACT. (b) Differential DMN activation during meditation instructions (BREATH) vs. a control condition (LOOK). Red colors indicate lower DMN activation during meditation instructions (BREATH) relative to a control condition (LOOK). Results significant at a whole brain threshold $(p<$ $0.001,>6$ voxels) and shown at $p<0.005$ for display purposes. BOLD - Blood-oxygen level-dependent; MTG - middle temporal gyrus; PCUN — precuneus; PHG - parahippocampal gyrus; CUN — cuneus; PCG - posterior cingulate cortex. X, y, and z are MNI peak coordinates.

\section{Hosted file}

Table_1_Meditation_reduces_brain_activity_in_the_default_mode_network_in_children_with_cancer.docx available at https://authorea.com/users/457771/articles/554485-meditation-reduces-brainactivity-in-the-default-mode-network-in-children-with-cancer

Hosted file 
Table_2_Meditation_reduces_brain_activity_in_the_default_mode_network_in_children_with_cancer.docx available at https://authorea.com/users/457771/articles/554485-meditation-reduces-brainactivity-in-the-default-mode-network-in-children-with-cancer

\section{Hosted file}

Table_3_Meditation_reduces_brain_activity_in_the_default_mode_network_in_children_with_cancer.docx available at https://authorea.com/users/457771/articles/554485-meditation-reduces-brainactivity-in-the-default-mode-network-in-children-with-cancer

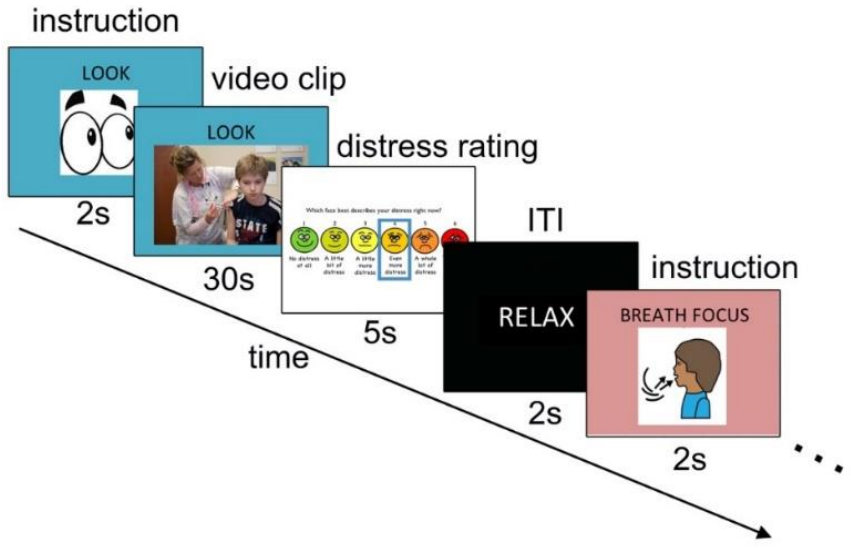

FIGURE 1 Emotion regulation task. Participants received one of four instructions prior to watching the video clip. After each clip, participants rated their level of distress $(1=$ no distress at all, $6=$ the worst possible distress) on a visual analog scale (VAS), which is an adapted version of the Wong and Baker's FACES scale. Instructions consisted of (1) LOOK, a control condition during which participants were instructed to passively view the video clip, (2) DISTRACT, a non-meditation condition during which participants were instructed to count backwards from ten, and two meditation emotion regulation techniques: (3) BREATH, during which participants were instructed to focus their attention on their breathing, and (4) ACCEPT, during which participants were instructed to pay attention to the emotions they were experiencing in a nonjudgement way. 


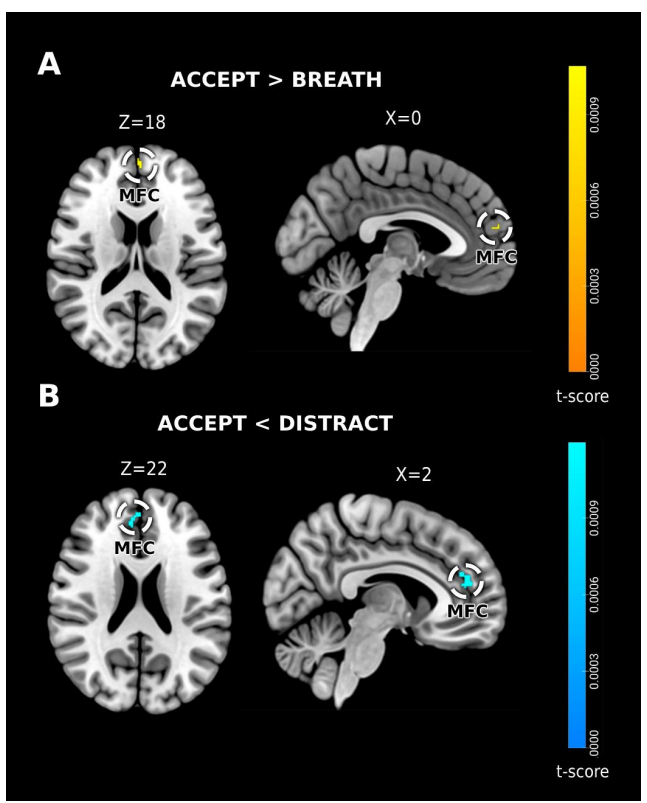

FIGURE 2 Region of interest results: Effects of meditation emotion regulation techniques on activation in the default mode network (DMN). (a) Differential DMN activation associated with ACCEPT vs. BREATH meditation instructions. Yellow colors indicate greater BOLD response in the DMN for ACCEPT vs. BREATH. (b) Differential DMN activation during meditation instructions (ACCEPT) vs. a non- meditation instruction (DISTRACT). Blue colors indicate lower DMN activation during meditation instructions (ACCEPT) relative to nonmeditation instructions (DISTRACT). Results significant at small-volume corrected threshold ( $p F W E \leq 0.05,>5$ voxels) and shown at $p<0.005$ for display purposes. BOLD - Bloodoxygen level-dependent; MFC - medial frontal cortex. X, y, and z are MNI peak coordinates. 


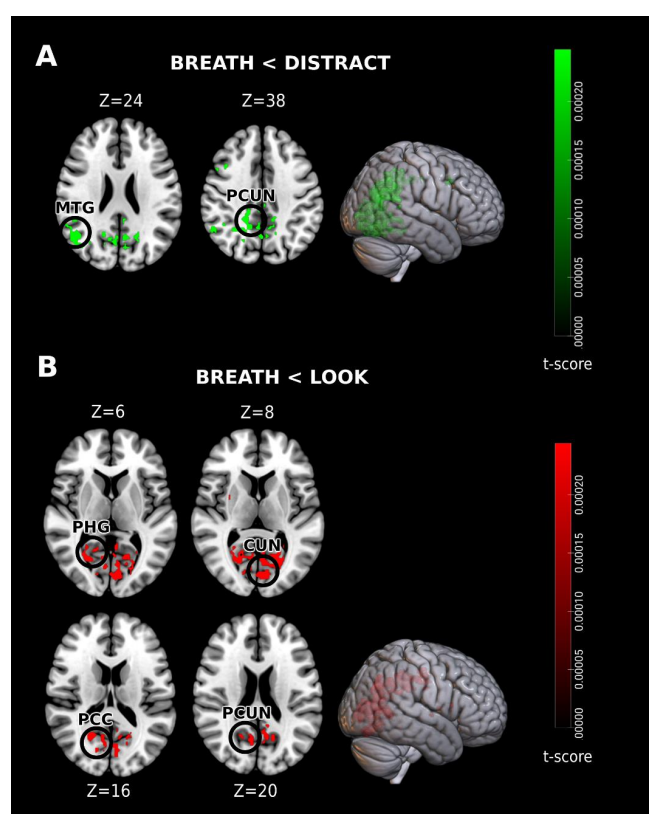

FIGURE 3 Whole brain results: Effects of meditation emotion regulation techniques on activation in the default mode network (DMN). (a) Differential DMN activation associated with meditation (BREATH) vs. non-meditation (DISTRACT) instructions. Green colors indicate lower BOLD response in the DMN for BREATH vs. DISTRACT. (b) Differential DMN activation during meditation instructions (BREATH) vs. a control condition (LOOK). Red colors indicate lower DMN activation during meditation instructions (BREATH) relative to a control condition (LOOK). Results significant at a whole brain threshold $(p<0.001,>6$ voxels $)$ and shown at $p<0.005$ for display purposes. BOLD - Blood-oxygen level-dependent; MTG — middle temporal gyrus; PCUN — precuneus; PHG — parahippocampal gyrus; CUN cuneus; PCG - posterior cingulate cortex. $\mathrm{X}, \mathrm{y}$, and $\mathrm{z}$ are MNI peak coordinates. 